\title{
BMJ Open Pedagogical strategies in teaching invasive prenatal procedures: a scoping review protocol
}

\author{
Gharid Nourallah Bekdache, ${ }^{\oplus 1}$ Maria Mylopoulos, ${ }^{2}$ \\ Kulamkan Mahan Kulasegaram, ${ }^{3}$ Rory Windrim ${ }^{4}$
}

To cite: Nourallah Bekdache G, Mylopoulos M, Kulasegaram KM, et al. Pedagogical strategies in teaching invasive prenatal procedures: a scoping review protocol. BMJ Open 2019;9:e024629. doi:10.1136/ bmjopen-2018-024629

- Prepublication history and additional material for this paper are available online. To view these files, please visit the journal online (http://dx.doi. org/10.1136/bmjopen-2018024629).

Received 7 June 2018

Revised 8 April 2019

Accepted 10 April 2019

Check for updates

(c) Author(s) (or their employer(s)) 2019. Re-use permitted under CC BY-NC. No commercial re-use. See rights and permissions. Published by BMJ.

${ }^{1}$ Maternal Fetal Medicine Division, Mount Sinai Hospital, Toronto, Ontario, Canada ${ }^{2}$ Wilson Centre, Undergraduate Medical Professions Education and Department of Paediatrics, University of Toronto, Toronto, Ontario, Canada

${ }^{3}$ Department of Family and Community Medicine and The Wilson Centre, University of Toronto and University Health Network, Toronto, Ontario, Canada

${ }^{4}$ Maternal Fetal Medicine Division, Mount Sinai Hospital, Toronto, Ontario, Canada

Correspondence to

Dr Gharid Nourallah Bekdache gbekdache@gmail.com

\section{ABSTRACT}

Introduction Invasive prenatal procedures (IPP) are core competencies in a Maternal-Fetal Medicine (MFM) fellowship training programme yet no standardised competency-based curriculum exists. This scoping review aims to provide a comprehensive understanding of the existing educational strategies for amniocentesis, chorionic villus sampling, fetal blood sampling and intrauterine blood transfusion. The objective is also to describe current gaps in the literature regarding evidencebased standards for training and assessment in IPP. Finally, we hope to encourage medical educators who are seeking to develop curricula based on competence by design to foster adaptive expertise through incorporating contextual variations in their teaching thus helping future MFM specialists to handle challenges and respond creatively to changing clinical circumstances and environmental variations.

Methods and analysis Using the five-stage framework of Arksey and 0'Malley's scoping review methodology as a guide, we will perform a systematic search in the Medline, Embase and Cochrane library databases to identify relevant studies on the educational strategies for IPP. We will include relevant English articles published after 1978. For a comprehensive search, we will explore websites and key journals, and hand-searched reference lists of key studies. Key studies are articles deemed relevant according to the specific inclusion and exclusion criteria. We will chart and sort data using a descriptive and thematic analysis approach.

Ethics and dissemination This review will be the first to examine all forms of pedagogical strategies used in training invasive fetal procedures. As an analysis of pre-existing available data in the literature, this scoping review does not require ethical approval. We anticipate that results will identify research gaps as well as novel ideas for education strategies and assessment. Findings from this study will be disseminated through publication in a peer-reviewed journal, medical education and clinical conferences, and in knowledge translation settings, aiming to improve clinical practice and quality of care.

\section{INTRODUCTION}

Procedural skills are an essential aspect of Maternal-Fetal Medicine (MFM) practice and an expected component of a MFM fellowship programme. Despite widespread ambitions

\section{Strengths and limitations of this study}

- This scoping review is a novel review approach offering an overarching picture of invasive fetal procedures, within the context of education and assessment.

- Findings will have implications for educators and researchers in recognising opportunities, pitfalls and contextual variations in teaching invasive prenatal procedure.

- This review comprises broad inclusion criteria (peer review journal and grey literature) without assessing the quality of the articles included which gives breadth and comprehensiveness and is consistent with scoping review guidelines.

- Rigour will be enhanced by a study design that includes the use of an established scoping review methodology, data-charting form and a transparent iterative team approach.

- Findings are limited to articles written in English.

to provide education by Competency BY Design (CBD) in many programmes, ${ }^{1}$ the rhetoric of CBD remains a wish more than practice for training MFM fellows in invasive prenatal procedures (IPP). Globally, MFM training programmes in IPP are developed by local experts who have traditionally learnt to perform these procedures in an apprenticeship model: 'see one, do one, and teach one'. ${ }^{2}$ This apprenticeship model is challenged by the significant reduction of the number of IPP due to the local and global implementation of non-invasive prenatal testing. ${ }^{3-7}$ Moreover, multiple external factors have reduced the opportunities for hands-on training in invasive procedures. These factors include centralisation, increasing staff workload and decreasing physician-teaching time. ${ }^{8}$ However, the widespread use of non-invasive prenatal testing has not completely replaced the role of IPP for diagnostic and therapeutic purposes. Thus, the need to find methods of effective training is now more pressing than ever. 
There are two important components to consider in this area: first, what are the appropriate frameworks for education in postgraduate medical education? Second, what are the current practices in training, and how do they align with known appropriate frameworks?

In terms of frameworks, two major ones have become prominent. The first framework is competency-based education in Canada with a specific variant known as competence by design (CBD). Competence by design is an outcome-based approach organised around competencies derived from an analysis of societal and patient needs to enhance patient safety and care by improving physician training and assessment. ${ }^{9}$ Much has been written about the advantages and disadvantages of this approach. We draw attention on essential limitations of CBD for training prenatal procedures. ${ }^{10}{ }^{11}$ One of the notable limitations is that standardisation does not address the challenges that future MFM practitioners will face in their independent practice. ${ }^{10}$ Outcome-based approach addresses routine cases through standardisation and algorithms. However, in practice, physicians must also be trained to be adaptable and address the challenges in non-routine and/or complex cases. ${ }^{10}{ }^{12-14}$ While CBD is useful for training procedural skills in strictly controlled environments, complementary frameworks may be needed to address training for complexity and novelty. One such framework is the adaptive expertise (AE) framework. This model of enhanced cognitive capacity supports learning when challenged by novel, complex or varied clinical presentations, and employ innovation and learning through practice. ${ }^{12-14} \mathrm{AE}$, a learnt skillset, develops over time and with practice ${ }^{101213}$ through a training environment that incorporates innovation, contextual variations, opportunities for students to struggle and immediate feedback. ${ }^{14}$ $\mathrm{AE}$ reduces the standardisation effect of competency-based education and enables future providers to be responsive to a constantly changing environment and dynamic environments. Moreover, the goal is not just to train innovators but those who can effectively combine routine practice as well as necessary innovation and adaptive problem solving by drawing on deep conceptual knowledge and wide arrays of skills. ${ }^{12-14}$ A combined framework of $\mathrm{CBD}$ and $\mathrm{AE}$ has been used previously to structure training for complex capacities and skills. ${ }^{15}$ The advantages of this approach for structuring curricula are also being established. While these frameworks are articulated in the general medical education literature, the alignment of existing practice in prenatal procedure training is less clear. A general overview of the existing literature did not show any consensus regarding the optimal pedagogical strategy or assessment that both addresses technical and non-technical skills in IPP when training adaptive experts. The initial review was limited to indications, ${ }^{5}$ outcomes, ${ }^{16} 17$ techniques,${ }^{18}$ complications ${ }^{3}$ and highlighted a lack of conceptual framework, and a paucity of evidence on the educational needs for future MFM fellows. ${ }^{14}$ Moreover, less is known about the clinical training environment including important questions on effective instructional and educational methods, assessments and feedback, and the structure of clinical training. Lastly, how these existing practices can lead to the production of competent and adaptive experts is unknown. In short, our overview lead us to suspect that a clear mapping of the literature is required.

To address this gap, we propose a scoping review in order to provide a 'snapshot' of current pedagogical approaches; to identify empirical evidence that informs medical educators and researchers regarding current practices in teaching IPP and to identify gaps in current knowledge. The primary objective of this research is to map any available evidence regarding techniques and education approaches in IPP, that is, available in both the peer-reviewed and grey literature. The second purpose is to examine how these approaches align and facilitate the future development of a competency-based curriculum in IPP that trains adaptive experts. Finally, the scoping review will contribute to the general IPP literature.

\section{PROTOCOL DESIGN}

To the authors' knowledge, there is no published knowledge synthesis of the pedagogical strategies used in teaching IPP. This makes a scoping review an important addition to this topic area. This scoping review follows the scoping review framework developed by Arksey and O'Malley, ${ }^{19}$ which has been enhanced further by Levac $e t$ $a l^{20}$ and the Joanna Briggs Institute (JBI). ${ }^{21}$ The results will be reported following the Preferred Reporting Items for Systematic Reviews and Meta-Analysis for Protocols guidelines. ${ }^{22}$ This method includes the following five steps: (1) identifying the research question; (2) identifying relevant studies balancing breadth and comprehensiveness; (3) study selection using an iterative team approach; (4) charting the data and (5) collating, summarising and reporting the results as they relate to the study purpose and implications of the study findings for policy, practice and research. The optional sixth step proposed by Arksey and O'Malley, ${ }^{19}$ which comprises a consultation with key stakeholders, will not be included in this report.

\section{Step1: identifying the research questions}

Based on our described objective, this primary review asks the following questions.

What educational interventions have been identified in the literature for teaching invasive prenatal procedure to MFM fellows?

In addition to this primary research question, we developed the following secondary research questions for this scoping review:

1. What type of research has been conducted in IPP and what were the goals?

2. What kind of educational activities that impart specific skills related to patient safety or healthcare quality are addressed in the literature? (Variety of educational strategies, teaching modalities used and so on) 
3. In what setting are these educational interventions provided?

4. What evidence, if any, is there of the effectiveness of these interventions?

5. What instruments have been developed and are being used to assess the learning outcomes for MFM fellows?

6. What are the barriers and the facilitators of education interventions identified in the literature?

7. What lesson is learnt as a result of this review?

\section{STEP 2: IDENTIFYING RELEVANT STUDIES}

A comprehensive review was developed with the help of an experienced health sciences librarian at the University of Toronto using specific Medical Subject Headings terms and keywords related to invasive prenatal procedures in order to review the relevant literature comprehensively (see online supplementary appendix 1). Studies or abstracts published from the year 1978, the year when ultrasound assistance was first introduced, until 2019 will be included. The search strategy will follow the three-step approach recommended by JBI scoping review guidelines. ${ }^{21}$ The search will be initially conducted using Ovid Medline electronic database and saved to ensure reproducibility of the search results. The Ovid interface is also a shared platform, which allows for quicker translation and querying of other Ovid-based databases (Medline In-Process, Embase) and will translate the search to Cochrane databases. Second, using all identified terms and keywords, we will supplement the search by a grey literature search utilising Google Scholar, Web of Science, websites of various relevant organisations, our institutional database, conference abstracts or reviews in order to identify any related studies. Grey literature is defined as 'Information produced on all levels of government, academia, business and industry in electronic and print formats not controlled by commercial publishing, i.e. where publication is not the primary activity of the producing body'. ${ }^{23}$ Finally, we will screen the bibliography of selected articles to identify articles relevant to this scoping review. We will frequently seek feedback from our research team to refine our search strategy, and we will contact authors of relevant primary studies or reviews for further information. We will also assess the quality of our search protocol using the PRESS 2015 Evidence-based checklist guidelines. ${ }^{24}$
All references will be imported in an online bibliographic management programme (RefWorks) ensuring removal of duplicates. We will also report the search strategy for the databases in the online supplementary appendix as outlined in a Preferred Reporting Items for Systematic Reviews and Meta-Analyses statement. ${ }^{25}$

\section{STEP 3: STUDY SELECTION}

An independent reviewer (GN) will apply a two-step approach screening to determine the eligibility of articles according to their inclusion and exclusion criteria. The eligibility criteria are developed in consensus by the research team and serve to filter for relevant sources. The first step will consist of a title and abstract scan and the second will entail a full-text review of all identified citations from step 1. A second reviewer (RW) will help to adjucicate uncertainty of the first reviewer about inclusion eligibility of specific titles and abstracts. A sample of the retrieved articles (ie, 20\%) will be screened by the second reviewer (RW) to ensure a consistent application of the eligibility criteria for inclusion in the review. Disagreements about study eligibility of the sampled articles will be discussed between the two reviewers until a consensus is reached and we will confer with a third reviewer if no agreement is reached.

\section{Inclusion criteria}

Based on 'Population-Concept-Context (PCC)' framework recommended by the JBI for scoping reviews ${ }^{21}$ and presented in table 1 .

\section{Exclusion}

1. We will exclude studies not providing a description of the instructional strategy, learning content and evaluation methods.

2. We will exclude studies representing opinions such editorials and commentaries.

\section{STEP 4: DATA CHARTING}

The research team will develop a data-charting form (table 2).

Since a scoping review aims to provide a comprehensive view of the literature, data extracted from relevant studies

\section{Table 1 PCC framework}

Population

Participants are any health personnel who provide health services within the provision of Obstetric and Maternal and Fetal Medicine healthcare during pregnancy and childbirth such as MFM specialists, MFM fellows, Obstetric Gynaecology residents and nurses

Concept Articles with specific focus and/or statements mentioning invasive prenatal procedural skills (amniocentesis, chorionic villus sampling, fetal blood sampling and intrauterine blood transfusion), competencies, assessments and educational activities conducted on human, animals and models

Context The review will include all study designs (qualitative, quantitative and mixed methods) as well as book chapters, published in English after 1978 when ultrasound was first introduced

MFM, Maternal-Fetal Medicine; PCC, Population-Concept-Context. 


$\begin{array}{ll}\text { Table } 2 \text { Draft-charting form } \\ \begin{array}{ll}\text { Study } & \text { First author last name } \\ \text { characteristics } & \text { Publication year } \\ & \text { Country } \\ & \text { Topic } \\ & \text { Purpose } \\ & \text { Publication type } \\ & \text { Study design } \\ & \text { Type of invasive prenatal procedure } \\ & \text { Curriculum } \\ \text { Educational } & \text { Training } \\ \text { component } & \text { Teaching } \\ & \text { Technique } \\ & \text { Assessment } \\ & \text { Mode of education delivery } \\ & \text { Competence by design } \\ \text { Conceptual } & \text { Adaptive expertise } \\ \text { or theoretical } \\ \text { framework }\end{array} & \text { Meaningful variation } \\ & \text { Preparation for future learning } \\ & \text { None } \\ & \text { Other }\end{array}$

$\begin{array}{ll}\text { Study setting } & \text { Clinical } \\ & \text { Classroom } \\ & \text { Simulation } \\ & \text { Online modules } \\ \text { Videos } \\ \text { Other }\end{array}$

Instructional Hands-on training

methods Competency based (mastery learning

and deliberate practice)

Step by step feedback

Unsupervised coaching

Reflective practices

Repetition: psychomotor training

$\begin{array}{ll}\text { Assessment } & \text { Checklists } \\ & \text { Feedback } \\ & \text { Global rating scale } \\ & \text { Direct observation } \\ & \text { Mini clinical evaluation exercise }\end{array}$

Major findings

will include general information about each article such as author, publication year, country, study purpose setting, methodology, outcomes, key findings and reported challenges and limitations. In addition, we will extract information specific to addressing the gaps in IPP education. Data will include the topic of the article, the type of the article (review, commentary, primary research), study design, study setting and an educational component (assessment, teaching, training and mode of delivery of teaching, training and assessment), conceptual or theoretical framework, instructional methods and assessment. The data-charting form will be refined during the fulltext screening to capture all pertinent information for each study. Articles that meet the eligibility criteria will be organised in data-charting form using Microsoft Excel database. Two reviewers (GN and RW) will pilot the data extraction form on a sample of three articles and will assess that the form identifies all different themes relevant to the research question.

\section{STEP 5: SYNTHESISING}

The fifth stage described by Arksey and O'Malley framework $^{19}$ for collating and summarising data will involve a descriptive numerical summary and a thematic analysis. We will use a qualitative thematic analysis approach to chart and sort the data according to key themes. This approach is a commonly used method for scoping review and it involves identifying themes across literature and synthesising using summary tables with thematic headings. ${ }^{26}$ We will summarise the quantitative data in a table outlining the overall number of studies, countries, topics, type and years of publication, and study designs. Next, we will organise, code and analyse the themes identified from all studies. The exact format cannot be established until data are charted and discussed with the review team.

\section{Patient and public involvement}

Patients and public were not involved in the study design.

\section{Ethics and dissemination}

This paper presents the protocol for a scoping review on the educational approaches in teaching IPP. The results from this study will inform clinicians, medical educators, knowledge users and researchers of the current gap in the literature and inform future study focused on the development of a standardised based curriculum.

Ethics approval is not necessary as the data are collected from publicly available sources and there will be no consultative phase. The results will be disseminated through presentations at local, national, and clinical and medical education conferences and through publication in a peer-reviewed journal.

Acknowledgements The authors would like to acknowledge the help of Gail Nichol (health research librarian at Gerstein Library, university of Toronto) for the design of the search strategy.

Contributors MM and KMK contributed to the project idea. MM, KMK and RW supervised the research. GNB contributed to the conceptual design of this review, conducted the literature review, the search strategy and drafted the protocol. MM and RW worked on editing. All authors approved the final manuscript. MM is the guarantor of the review.

Funding The authors have not declared a specific grant for this research from any funding agency in the public, commercial or not-for-profit sectors.

Disclaimer The views expressed in this study reflects the authors' own perspectives.

Competing interests None declared.

Patient consent for publication Not required.

Provenance and peer review Not commissioned; externally peer reviewed.

Open access This is an open access article distributed in accordance with the Creative Commons Attribution Non Commercial (CC BY-NC 4.0) license, which permits others to distribute, remix, adapt, build upon this work non-commercially, and license their derivative works on different terms, provided the original work is properly cited, appropriate credit is given, any changes made indicated, and the use is non-commercial. See: http://creativecommons.org/licenses/by-nc/4.0/.

\section{REFERENCES}

1. Busing N, Harris K, MacLellan AM, et al. The future of postgraduate medical education in Canada. Acad Med 2015;90:1258-63. 
2. Cameron JL. William Stewart Halsted. Our surgical heritage. Ann Surg 1997;225:445-58.

3. Zwiers C, Lindenburg ITM, Klumper FJ, et al. Complications of intrauterine intravascular blood transfusion: lessons learned after 1678 procedures. Ultrasound Obstet Gynecol 2017;50:180-6.

4. Dalton SE, Gregg AR, Ho M. Second-trimester uterine model for teaching ultrasound-guided obstetric procedures. J Ultrasound Med 2017;36:1723-31.

5. Lindenburg IT, van Kamp IL, Oepkes D. Intrauterine blood transfusion: current indications and associated risks. Fetal Diagn Ther 2014;36:263-71.

6. Lichtenbelt KD, Alizadeh BZ, Scheffer PG, et al. Trends in the utilization of invasive prenatal diagnosis in The Netherlands during 2000-2009. Prenat Diagn 2011;31:765-72.

7. Uhlmann RA, Taylor M, Meyer NL, et al. Fetal transfusion: the spectrum of clinical research in the past year. Curr Opin Obstet Gynecol 2010;22:155-8.

8. Hui L, Tabor A, Walker SP, et al. How to safeguard competency and training in invasive prenatal diagnosis: 'the elephant in the room'. Ultrasound Obstet Gynecol 2016;47:8-13.

9. Frank JR, Mungroo R, Ahmad Y, et al. Toward a definition of competency-based education in medicine: a systematic review of published definitions. Med Teach 2010;32:631-7.

10. Cutrer WB, Ehrenfeld JM. Protocolization, standardization and the need for adaptive expertise in our medical systems. J Med Syst 2017;41:200.

11. Pinsk M, Karpinski J, Carlisle E. Introduction of Competence by Design to Canadian Nephrology Postgraduate Training. Can J Kidney Health Dis 2018;5:205435811878697.

12. Mylopoulos M, Kulasegaram K, Woods N. Developing the experts we need: Fostering adaptive expertise through education. Health \& Medicine Week 2018.

13. Mylopoulos M, Woods NN. Having our cake and eating it too: seeking the best of both worlds in expertise research. Med Educ 2009;43:406-13.
14. Mylopoulos M, Steenhof N, Kaushal A, et al. Twelve tips for designing curricula that support the development of adaptive expertise. Med Teach 2018;40

15. Sockalingam S, Mulsant BH, Mylopoulos M. Beyond integrated care competencies: The imperative for adaptive expertise. Gen Hosp Psychiatry 2016;43:30-1.

16. Alfirevic Z, Navaratnam K, Mujezinovic F. Amniocentesis and chorionic villus sampling for prenatal diagnosis. Cochrane Database Syst Rev 2017;13.

17. Tongprasert F, Wanapirak C, Sirichotiyakul S, et al. Training in cordocentesis: the first 50 case experience with and without a cordocentesis training model. Prenat Diagn 2010;30:30.

18. Dodd JM, Windrim RC, van Kamp IL. Techniques of intrauterine fetal transfusion for women with red-cell isoimmunisation for improving health outcomes. Cochrane Database Syst Rev 2012;9:CD007096.

19. Arksey H, O'Malley L. Scoping studies: towards a methodological framework. Int J Soc Res Methodol 2005;8:19-32.

20. Levac D, Colquhoun $\mathrm{H}, \mathrm{O}^{\prime}$ Brien KK. Scoping studies: advancing the methodology. Implement Sci 2010;5:69.

21. The Joanna Briggs Institute. Joanna briggs institute reviewers'manual 2015. 2015 https:// joannabriggs.org/ assets/ docs/ sumari/Reviewers- Manual_Methodology-for- JBI- ScopingReviews 2015 v2.pdf.

22. Moher D, Liberati A, Tetzlaff J, et al. Preferred reporting items for systematic reviews and meta-analyses: the PRISMA Statement. Open Med 2009;3:e123-30.

23. Farace D, Schöpfel J, eds. Grey Literature in Library and Information Studies. Berlin: De Gruyter Saur, 2010.

24. McGowan J, Sampson M, Salzwedel DM, et al. PRESS Peer Review of Electronic Search Strategies: 2015 guideline statement. J Clin Epidemiol 2016;75:40-6.

25. Shamseer L, Moher D, Clarke M, et al. Preferred reporting items for systematic review and meta-analysis protocols (PRISMA-P) 2015 elaboration and explanation. BMJ 2015;349:g7647.

26. Kastner M, Tricco AC, Soobiah C, et al. What is the most appropriate knowledge synthesis method to conduct a review? Protocol for a scoping review. BMC Med Res Methodol 2012;12:114. 